Supplementary information for

\title{
How alcoholic disinfectants affect coronavirus model membranes: membrane fluidity, permeability, and disintegration
}

Hossein Eslami, ${ }^{*}, 1,2$ Shubhadip Das, ${ }^{1}$ Tianhang Zhou, ${ }^{1}$ Florian Müller-Plathe ${ }^{1}$

${ }^{1}$ Eduard-Zintl-Institut für Anorganische und Physikalische Chemie, Technische Universität

Darmstadt, Alarich-Weiss-Str. 8, 64287 Darmstadt, Germany

${ }^{2}$ Department of Chemistry, College of Sciences, Persian Gulf University, Boushehr 75168, Iran

${ }^{*}$ Corresponding author.

Email: h.eslami@theo.chemie.tu-darmstadt.de 


\section{Ethanol Penetration into the Lipid Bilayer at 298 K}

In Figure S1 we have shown snapshots of simulation box for NPT simulations of DPPC (at 298 $\mathrm{K}$ ) immersed in aqueous solutions containing $0 \mathrm{~mol} \%, 5.0 \mathrm{~mol} \%, 9.6 \mathrm{~mol} \%$, and $17.5 \mathrm{~mol} \%$ ethanol. Compared to the liquid crystalline phase of the DPPC (see the main text), ethanol does not noticeably penetrates into the membrane.

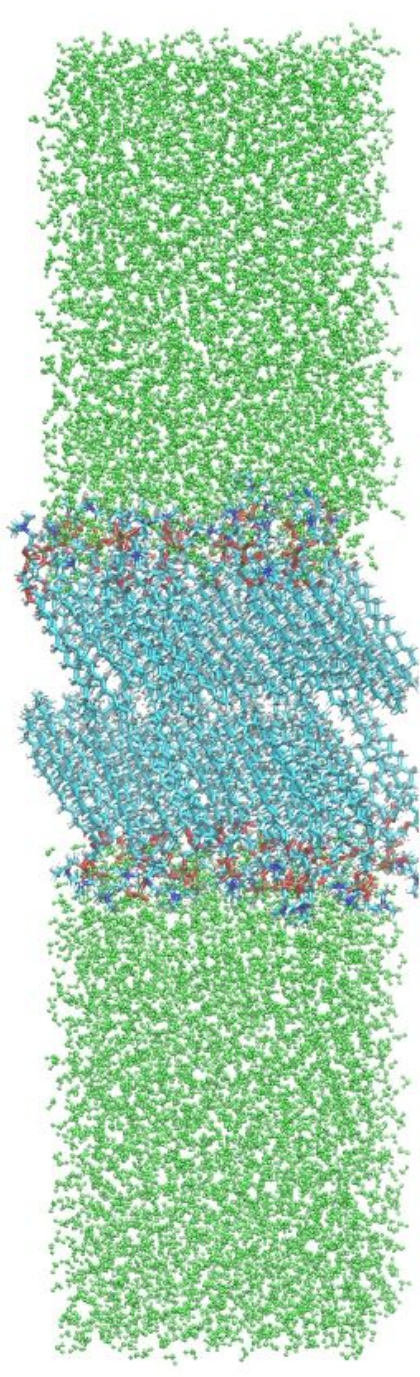

(a)

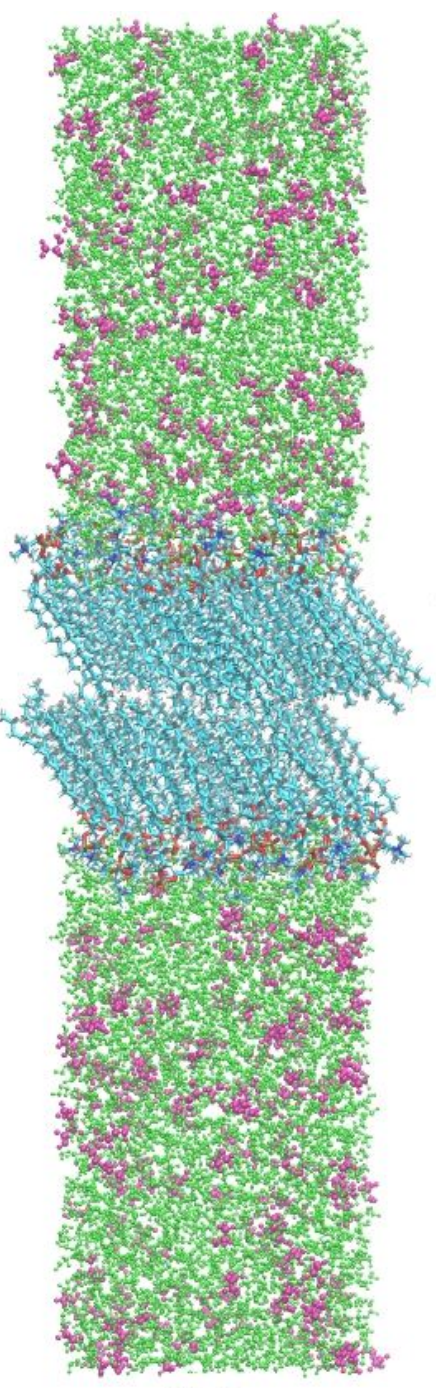

(b)

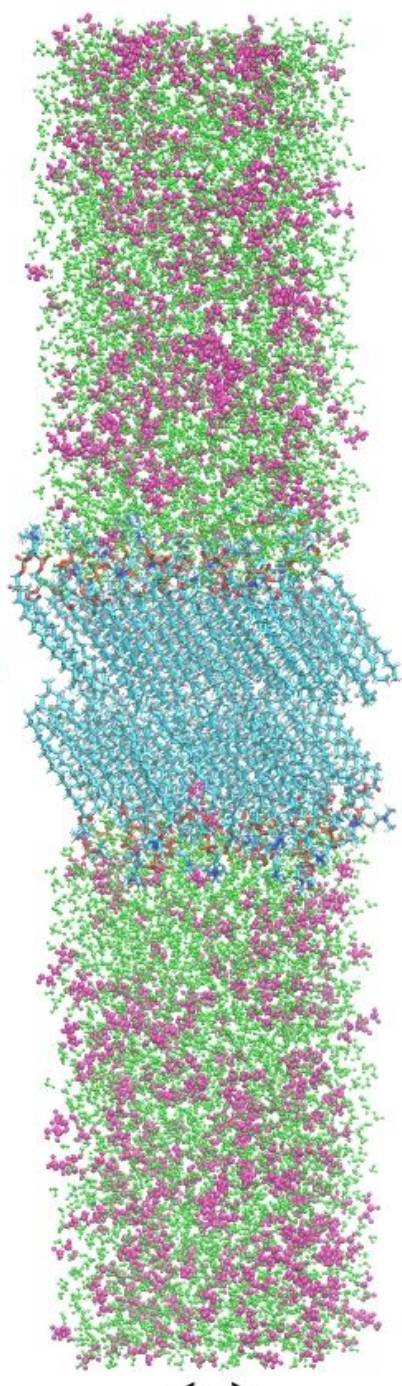

(c)

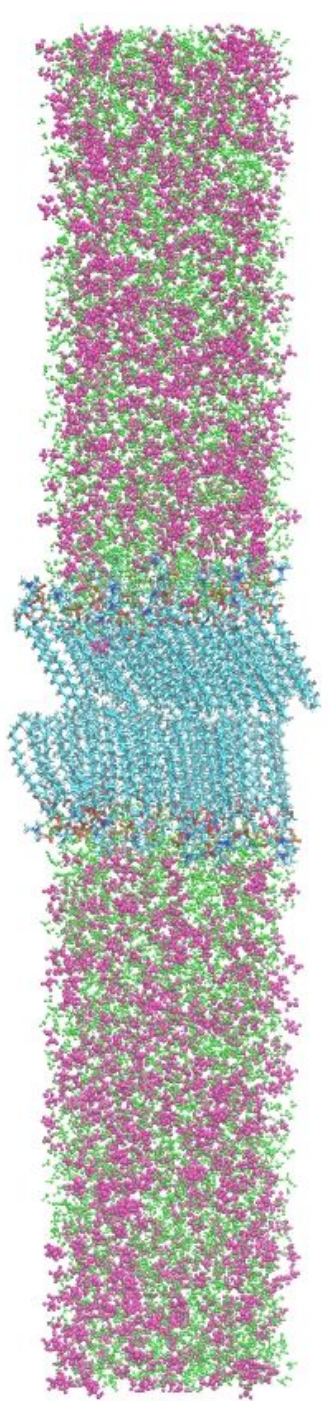

(d)

Figure S1. Snapshots of simulation box for DPPC immersed in 0, 5.0, 9.6, and $17.5 \mathrm{~mol} \%$ ethanol solutions (from a to d, respectively) at $298 \mathrm{~K}$. The water and ethanol molecules are shown in green and purple, respectively. All snapshots are taken at $t=750 \mathrm{~ns}$. 


\section{Effect of Ethanol on the Structure of the Lipid Bilayer}

Figure S2 indicates the density profiles for lipid chains of each leaflet in the absence and presence of ethanol at $298 \mathrm{~K}$. Compared to the liquid crystalline phase of membrane (see the main text) no noticeable effect of alcohol on the lipid phase (interdigitation of lipid tails) is observed in the gel phase of DPPC.

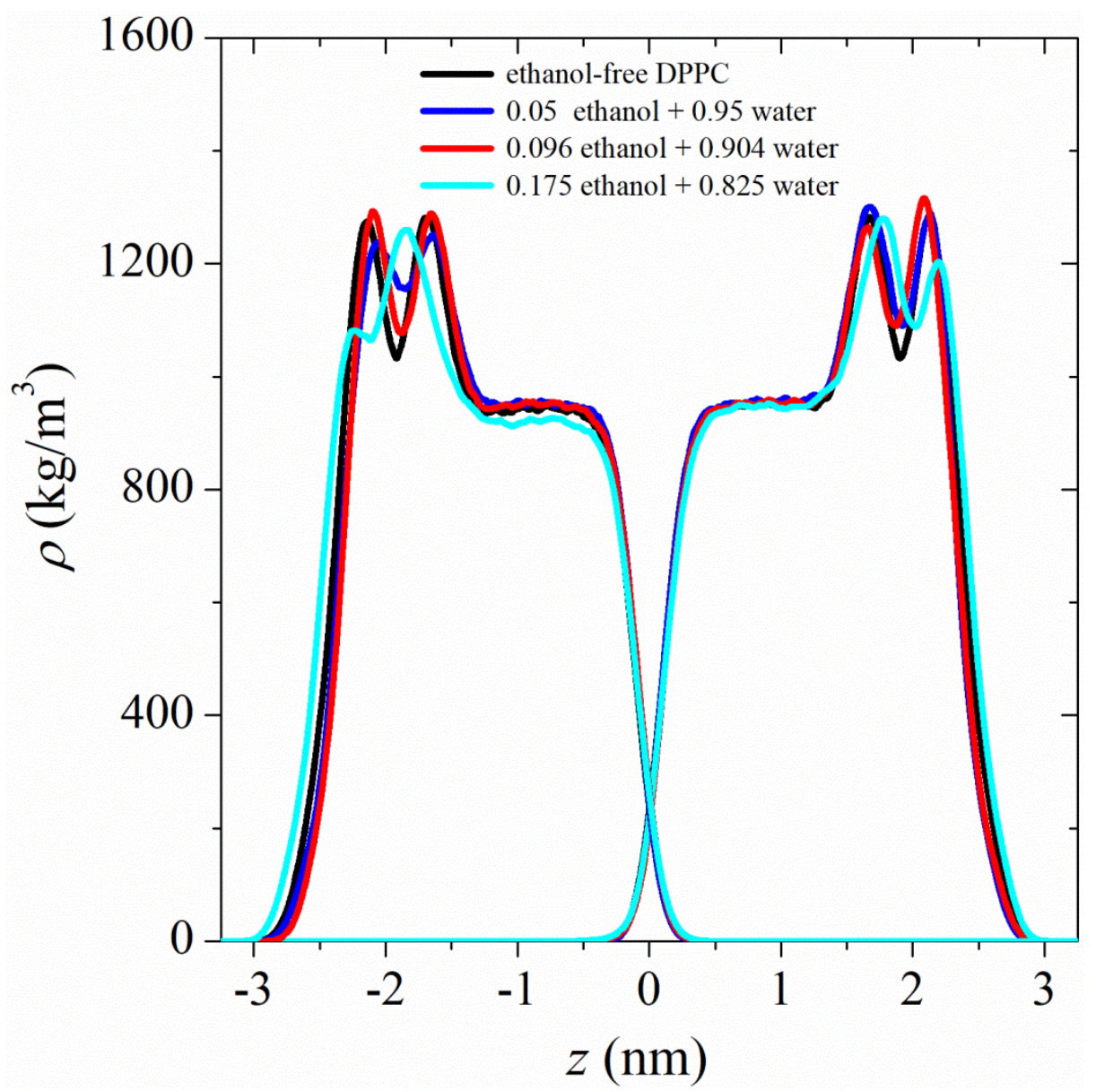

Figure S2. Density profiles for lipid chains of two opposite leaflets at $298 \mathrm{~K}$. 